\section{$262 \quad$ VENTRICULAR DYSFUNCTION PRECEDES CARDIAC HYPERTROPHY IN RESPONSE TO ENHANCED ANGIOTENSIN II TYPE 1 RECEPTOR ACTIVITY IN THE DIFFERENTIATED CARDIOMYOCYTE}

G A Frentzou, M J Drinkhill, N A Turner, S G Ball, J F X Ainscough University of Leeds

doi:10.1136/heartjnl-2013-304019.262

Left ventricular hypertrophy (LVH) is frequently associated with elevated blood pressure and ventricular dysfunction in response to modulation of the renin-angiotensin system (RAS). Ultimately this leads to pathological remodelling with characteristic cardiomyocyte death and fibrosis, resulting in heart failure. We have created a conditional transgenic mouse model in which a mutant Human Angiotensin II type I Receptor Transgene (HART) can be activated exclusively in the differentiated cardiomyocyte. Twelve-month old HART mice exhibit characteristics relevant to clinical LVH, with ventricular dysfunction, significant cardiomyocyte hypertrophy and subsequent interstitial fibrosis upon stimulation of the transgenic receptor, without increase in blood pressure.

In this study we have utilised the HART activation system in adults at 5 months old to begin to unravel cellular and molecular mechanisms involved in stimulating the induction and subsequent progression of LVH. Notably, the HART mice exhibit significant ventricular dysfunction at this early stage, with no evidence of hypertrophy at either the individual cardiomyocyte or whole heart level. Moreover, there was no evidence of fibrosis or increased cardiomyocyte death that may account for the observed dysfunction.

Stimulation of the transgenic receptors with Ang IV for 4 weeks induced LVH with worsening ventricular function, but only limited evidence of further pathological remodelling. However, TUNEL assay suggested an increase in number of dying myocytes in otherwise 'healthy' regions of the myocardium. Furthermore, it was observed that TGF $\beta$ expression, previously reported to be up-regulated in LVH, was significantly decreased in the myocytes of transgenic animals treated with Ang IV, as opposed to the nonmyocyte population where TGF $\beta$ expression remained unchanged. Small sporadic areas of fibrosis were noted in some of the Ang IV exposed hearts, characterised by an increased density of nonmyocyte cells undergoing increased cell turnover. This nonmyocyte population demonstrated a significant increase in VEGF $\alpha$ expression whereas VEGF $\alpha$ levels remained un-changed in the myocytes.

Our results demonstrate that activation of RAS in cardiomyocytes leads directly to dilated cardiomyopathy without causing cardiomyocyte hypertrophy or death, demonstrating an initiating role for the cardiomyocyte in driving dysfunction. Further sustained stimulation of RAS in adult cardiomyocytes stimulates sporadic remodelling through hypertrophy and death of individual cardiomyocytes, closely associated with infiltration, activation and/or proliferation of non-myocyte cells (fibroblast and inflammatory). This secondary response involves activation of non-myocyte specific compensatory mechanisms, including an increase in angiogenic signalling. 